\title{
A Comparison of Nosocomial Infection Density in Intensive Care Units on Relocating to a New Hospital
}

\author{
Zeynep Ture ${ }^{*}$, Tugba Ustuner², Ario Santini ${ }^{3}$, Serhat Aydogan², IIhami Celik² \\ 1 Erciyes University Faculty of Medicine Kayseri, Turkey \\ 2 City Hospital of Kayseri, Turkey \\ 3 George Emil Palade University of Medicine, Pharmacy, Science, and Technology of Targu Mures, Romania
}

\begin{abstract}
Background: The study aimed to investigate the changes in nosocomial infection density after patients were transferred to the intensive care unit (ICU) of a new-build hospital. Methods: The types and rates of nosocomial infections were obtained for a one-year period retrospectively before leaving the old hospital premises and for a one-year periods after moving into the new hospital. The intensive care unit in the "old" premises was comprised of a 17-bedded hall, and thirty-three nurses shifted to work forty-eight hours a week, with each nurse assigned to provide care for two patients. The intensive care unit in the "new" premises consisted of single rooms, each with twenty-eight beds. Results: The median nosocomial infection density decreased from 23 to 15 per 1000 in-patient days. The catheterrelated urinary tract infection rate decreased from 7.5 to 2.6 per100 catheter days. Conclusions: Treatment of patients in the new hospital resulted in a decrease in nosocomial infection density.
\end{abstract}

Keywords: nosocomial infection density, infection control measures, causative microorganisms, antimicrobial susceptibility

Received: 12 February 2020 / Accepted: 21 June 2020

\section{INTRODUCTION}

Nosocomial infections are important causes of mortality, especially in intensive care units (ICU) [1]. Multidrug-resistant microorganisms may cause an increase in treatment costs and hospital stay [2,3]. Appropriate antibiotic combinations and doses are not known especially for the pan-drug resistant strains of Gram-negative bacteria [4] and the discovery and development of new antibiotics against carbapenem-resistant Klebsiella pneumoniae, Acinetobacter baumannii, and Pseudomonas aeruginosa have been determined as primary targets by WHO (2017) [5].

Colonisation with resistant microorganisms begins 48-72 hours after hospitalisation [6]. It is essential to take appropriate isolation measures and to monitor infected or colonised patients with resistant microorganisms in single rooms. It is assumed that healthcare workers (HCW) pay more attention to hand hygiene compliance when treating patients hospitalised in single rooms, and this leads to a decrease in the crosstransport of resistant microorganisms [7].
The first step of the isolation procedures is to initiate a staff hand-hygiene regime before patient contact, before undertaking an aseptic task, after exposure to bodily fluids, after patient contact and after contact with a patient surrounding $[8,9]$. The mechanism hypothesised for fewer infections in single rooms is the reduction of person-to-person contacts as well as limiting the spread of infection by person-surface-person contacts [10].

In May 2018, The Education and Research Hospital $(\mathrm{OldH})$ was closed, and all facilities relocated to a new hospital (NewH), The City Hospital, Kayseri, Turkey. The NewH had 1600 rooms, each with 248 beds including an ICU and dedicated resuscitation facility. The $\mathrm{NewH}$ was comprised of four towers. One tower, which included a 28-bed anaesthesia ICU, could accommodate 480 patients. This compared with the $\mathrm{OldH}$ which had 700-bed capacity with 17 beds ( 13 of the beds did not have a separated room), including an ICU.

The design of the intensive care units, infection care practices and staffing responsibilities in the ICUs of the 
OldH versus the NewH are detailed in Appendix 1.

The study aimed to investigate if there was a change in the number of nosocomial infections and infection density, causative microorganisms and antimicrobial susceptibility between the Old Hospital and the new hospital.

The null hypotheses are:

- There was no change in the number of nosocomial infections and infection density between the Old Hospital and the new hospital.

- There was no change in the types of causative microorganisms between the Old Hospital and the new hospital.

- There was no change in antimicrobial susceptibility between the Old Hospital and the new hospital.

\section{MethOD}

Data relating to treatment provided from 1 ${ }^{\text {st }}$ May 2017 to $30^{\text {th }}$ April 2017, was collected from the Education and Research Hospital (OldH) files, and from $1^{\text {st }}$ May 2018 to $30^{\text {th }}$ April 2019 from files in The City Hospital (NewH).

These included patients' age, gender and co-morbidities from the hospital laboratory information.

The types and rates of nosocomial infections, causative microorganisms and antimicrobial susceptibility during the study period were obtained from the records of the Infection Control Committee in both hospitals.

\section{Inclusion criteria of the patients in both hospitals}

- Patients diagnosed with ventilator-associated pneumonia, catheter-related bloodstream infection, urinary tract infection, and surgical site infection

- Patients who were treated with at least 72 hours of antibiotic treatment

\section{Exclusion Criteria of the patients in both hospitals}

- Patients whose demographic information is not available according to hospital medical records and infection control committee records.

- Patients $<18$ years

The study was ethically approved with the number of 2019-610 by University of Erciyes Medical Faculty.

\section{Statistical analysis}

The statistical analysis was performed using SPSS software version 22 (Chicago, IL, USA). The Chi-squared test was used for the categorical variables. MannWhitney $U$ test was used to determine the differences between the OldH and NewH. The level of significance was set at $\alpha=0.05$ for all tests.

\section{RESULTS}

During the study periods, 1210 patients were treated in the OldH, and 1152 patients in $\mathrm{NewH}$ The mean and standard deviation [mean (SD)] age of patients was 64 (10.1) in the OldH and 63 (13.7) in the NewH. The percentage of female patients was $45 \%$ and $60 \%$ respectively. The percentage of male patients was $55 \%$ and $40 \%$ respectively (Table 1). There was no difference between the patients in terms of co-morbidity (Table 1).

In the study periods, 127 nosocomial infection attacks were recorded in the OldH, while 96 attacks were recorded in the NewH. There was a statistically significant decrease in the median nosocomial infection density, decreased from 23 patient days per 1000 in the OldH to 15 patient days per 1000 in the NewH in-patient days (Mann- Whitney U test; $p=0,040$ ) (Table 2). The first Null hypothesis is partially rejected.

Overall there was no change in the types of causative microorganisms between the OldH and the $\mathrm{NewH}$ (Figure 1). The second null hypothesis is, therefore upheld.

Antimicrobial susceptibility of A. baumannii was similar in the OldH and NewH. The carbapenem resistance rate was above $95 \%$ in both hospitals. There was

Table 1. Comparison of demographic data and co-morbidities of patients in the old hospital (OldH) and the new hospital (NewH)

\begin{tabular}{lcccc} 
& $\begin{array}{c}\text { OldH } \\
\text { n=127 }\end{array}$ & $\begin{array}{c}\text { NewH } \\
\text { n=96 }\end{array}$ & $\begin{array}{c}\text { P } \\
\text { Chi-squared } \\
\text { test }\end{array}$ \\
Age (mean SD ) & 64.9 & 63.9 & $0.949^{*}$ \\
Female (\%) & $(10.1)$ & $(13.7)$ & $0.088^{*}$ \\
Male \% & 45 & 60 & $0.079^{*}$ \\
CHF (number) & 55 & 40 & $0.748^{*}$ \\
DM (number) & 5 & 8 & $0.748^{*}$ \\
CE (number) & 6 & 5 & $0.270^{*}$ \\
CRF (number) & 11 & 5 & $0.065^{*}$ \\
Trauma (number) & 5 & 4 & $0.652^{*}$ \\
Malignancy (number) & 9 & 7 & $0.076^{*}$ \\
Hemodialysis (number) & 9 & 3 & $0.847^{*}$ \\
\hline * No Statistical significance. OldH, old hospital; NewH, new hospital; CHF, Congestive Heart Failure;
\end{tabular}

DM, Diabetes Mellitus; CE, Cerebrovascular Events; CRF, Chronic Renal Failure 
Table 2. Comparisons of the number of nosocomial infections and infection density, in the old hospital (OldH) and new hospital (NewH)

\begin{tabular}{lccc} 
& OldH & NewH & P Mann- Whitney U test \\
Number of Nosocomial Infections & 127 & 96 & 0.401 \\
Nosocomial Infection Density & $23.0(16.5-46.4)$ & $15.0(4.1-32.5)$ & $0.040^{*}$ \\
\hline
\end{tabular}

$\mathrm{OldH}$, old hospital; NewH, new hospital. * Significant difference.

ICU of the OldH

ICU of the NewH

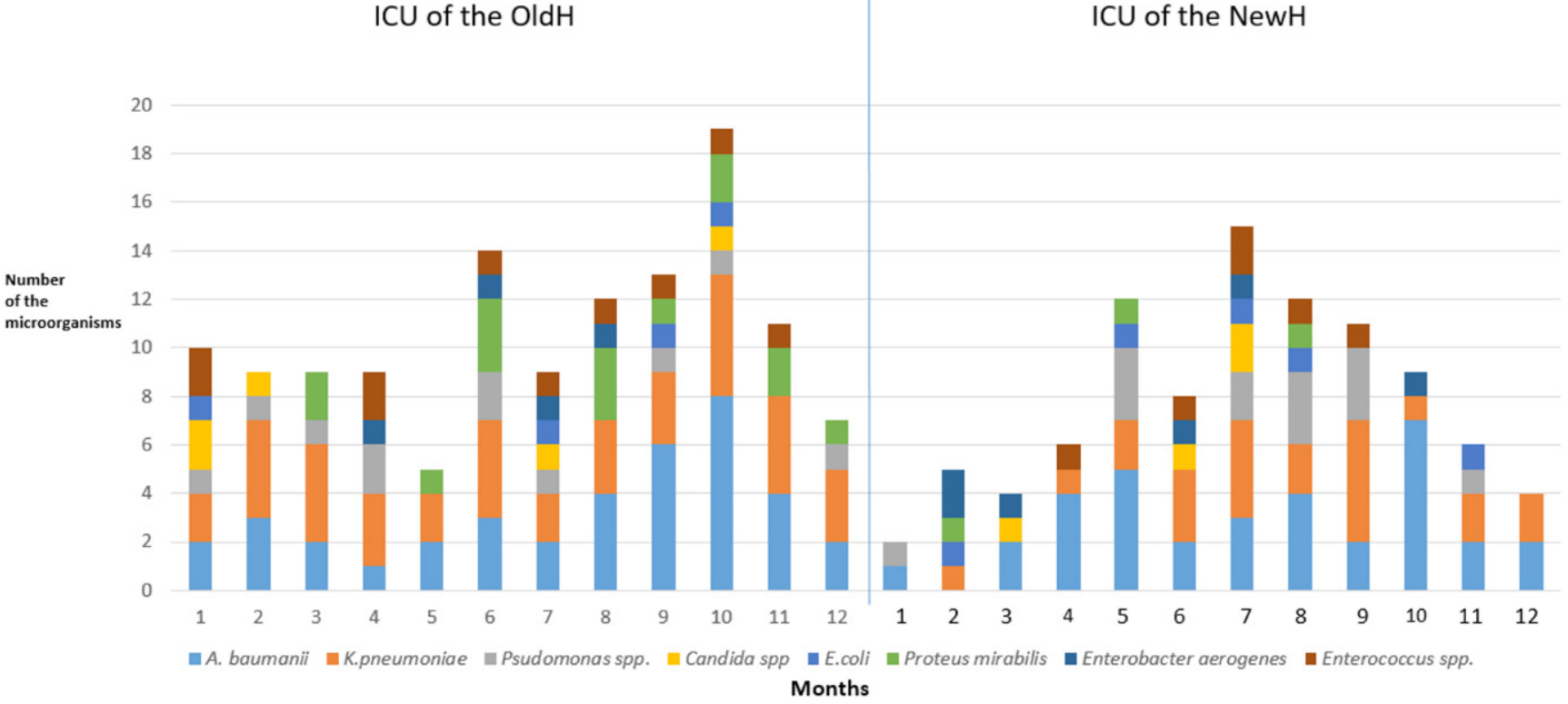

Fig. 1. The types and numbers of causative microorganisms associated with nosocomial infections in the two hospitals.

no significant difference between the two hospitals in terms of antimicrobial susceptibility of gram-negative microorganisms (Table 3). The third Null hypothesis is, therefore upheld.

The following data referring to secondary objectives were obtained. The rate of ventilator usage was 0.32 in the OldH and was 0.29 in the NewH (Mann- Whitney $\mathrm{U}$ test; $p=0.101$ ). Ventilator-Associated Pneumonia (VAP) rate decreased from 15.2 to 12.5 in 1000 ventilator days (Mann- Whitney $U$ test; $\mathrm{p}=0.438$ ). Although urinary catheters were used in $100 \%$ of patients in the $\mathrm{NewH}$, this compared to $93 \%$ of patients had urinary catheters in the OldH (Mann- Whitney U test; $p=$ 0.005).

The catheter-associated urinary tract infection (CAUTI) rate decreased from 7.5 to 2.6 per 100 catheter days between the OldH and the NewH. This was significantly different (Mann- Whitney U test; $p=0,047$ ).
There was no significant difference between the two hospitals in terms of central venous catheter usage rate (Mann- Whitney U test; $p=0.243$ ) and central catheterrelated bloodstream infection rate (Mann- Whitney $\mathrm{U}$ test; $p=0.748$ )

\section{DISCUSSION}

There was no difference in terms of co-morbidity between the patients treated in the $\mathrm{OldH}$ versus those treated in the NewH. There was no significant difference between two hospitals in terms of antimicrobial susceptibility of gram-negative microorganisms, nor was there a significant difference in the number of nosocomial infections on transferring patients to the $\mathrm{NewH}$. However, there was a significant difference in infection density. The need for rapid access to essential materials is stated in the optimal design of ICU's [11].

Table 3. Comparisons of the antimicrobial susceptibility, in the old hospital (OldH) and new hospital (NewH)

\begin{tabular}{lccc} 
& OldH (n=127) & NewH (n=96) & P Mann- Whitney U test \\
Carbapenem resistant Acinetobacter baumannii & $34(26 \%)$ & $30(31 \%)$ & $0.847^{*}$ \\
Carbapenem resistant Klebsiella pneumoniae & $30(23 \%)$ & $20(21 \%)$ & $0.056^{*}$ \\
Carbapenem resistant Pseudomonas aeruginosa & $9(7 \%)$ & $12(12,5 \%)$ & $0.949 *$ \\
\hline Old old hospital: NewH, new hospital. *No significant difference. & &
\end{tabular}


The most important of these are hand washing units and hand disinfectants. In this study, there was a significant increase in the number of handwashing units and hand disinfectants and access points. Increased compliance with hand hygiene may lead to a decrease in the density of nosocomial infections due to VRE, methicillin-resistant Staphylococcus aureus (MRSA), and multi-drug resistant Gram-negatives [12,13]. These parameters may have accounted for the reduction of nosocomial infection density.

Despite the increase in the number of beds in the ICU of the NewH, the number of nurses did not increase. Also, there was a threefold increase in the total working area in a shift. Both parameters are important factors for the increase of total workload, and compliance with infection control measures may decrease [11]. Increases in catheter-related urinary tract infection rate with increased workload have also been reported previously [14]. While the nurse-patient ratio was 0.5 in the 24 -hour period in the old ICU, it decreased to 0.4 in the new ICU. Both rates are not suitable for ergonomic patient care. In a prospective study evaluating 10657 patient days and 415 nosocomial infections in the ICU, increasing the nurse / patient ratio above 2.2 reported a $29 \%$ reduction in nosocomial infection density [15]. In a survey conducted with the nurses, it was reported that compliance with hand hygiene procedures decreased with high workload [16].

In this study, another compelling factor in decreasing the density of nosocomial infection was thought to be the change in the type of intensive care unit. Similar to the present study, an 11-year retrospective study of nosocomial infectious agents before and after moving to a single-room ICU reported a significant reduction in the number of multidrug-resistant microorganisms. Also, when clonal evaluation of the microorganisms in the old and new hospitals was performed, the clonal affinity of the multi-drug resistant gram-negative bacteria isolated in the old hospital was determined and spread by cross transport [7]. In a 24-bed ICU where nosocomial infections and colonisation were evaluated before and after the four years of single room design; 47\% reduction in MRSA, 43\% decrease in Clostridium difficile and 51\% decrease in yeasts were reported [17]. In the present study, although there is no change in the resistance profile of the causative microorganisms, nosocomial infection rates were predicted to decrease with the correction of other conditions.
Urinary catheter utilisation rate increased in the ICU of the NewH; however, a significant decrease was observed in catheter-related urinary tract infection rates. Non-compliance with infection control measures during catheter administration and catheter care after administration is among the most important risk factors for CAUTI [8]. After moving to the new hospital, not only ICUs but also emergency room and operating room conditions have been improved. Considering that urinary catheter applications are generally performed in these departments in patients admitted to ICU, it may contribute to a decrease in infection rates. Barbadoro et al. (2015) reported in a 2-year surveillance study [18] that performing urinary catheter administration outside the operating room increased the risk of CAUTI by 7.9 times. A review of nineteen studies reported that CAUTI decreased with compliance with hand hygiene during the urethral catheter administration and catheter care [19]. After relocation, improvement of hygienic conditions in all units of the new hospital, contributed, directly and indirectly, to decrease nosocomial infection rates in the ICU.

There was a decrease in the rate of ventilator usage and the rate of VAP; in both periods, the rate of VAP is still higher [20]. The VAP rate of the hospital is compatible with seventy-five percentiles according to the country average [21]. The main pathogenic factor in the development of VAP is biofilm formation within the endotracheal tube and microaspiration of secretions [22]. The incidence of VAP can be reduced by several means, including the use of care bundles [22]. Some of the bundle parameters include head-of-bed elevation, daily sedation vacation, daily assessment of readiness for extubation, prophylaxis of peptic ulcer and deep vein thrombosis, oral care with chlorhexidine solution, in-line suction system and subglottic suctioning [23]. In order to decrease the VAP rates, the first step should be reached the sufficient number of HCW, then would be to start a bundle application by using the appropriate ones from these parameters.

\section{CONCLUSION}

On relocating to a new hospital, though there was no change in the number or types of nosocomial infections, there was a decrease in the density of nosocomial infections. This may in part be due to the increase in the number of single-room facilities, hand washing units and the convenient availability of hand disinfectant. 


\section{CONFLICT OF INTEREST}

None to declare.

\section{REFERENCES}

1. Gao B, Li X, Yang F, et al. Molecular Epidemiology and Risk Factors of Ventilator-Associated Pneumonia Infection Caused by Carbapenem-Resistant Enterobacteriaceae. Front Pharmacol. 2019;10:262.

2. Giraldi G, Montesano M, Frati P, et al. Healthcare-Associated Infections Due to Multidrug-Resistant Organisms: a Surveillance Study on Extra Hospital Stay and Direct Costs". Current Pharmaceutical Biotechnology.2019; 20: 1.

3. Sosa-Hernández $O$, Matías-Téllez B, Estrada-Hernández A, Cureño-Díaz MA, Bello-López JM. Incidence and costs of ventilator-associated pneumonia in the adult intensive care unit of a tertiary referral hospital in Mexico. Am J Infect Control. 2019; 11.

4. Trecarichi EM, Tumbarello M. Therapeutic options for carbapenem-resistant Enterobacteriaceae infections. Virulence. 2017;8(4):470-484.

5. www.who.int/medicines/publications/global-priority-listantibiotic-resistant-bacteria/en/. Available date: 17.04.2019.

6. Levy Hara G, Kanj SS, Pagani L, Abbo L, Endimiani A, Wertheim HF. Ten key points for the appropriate use of antibiotics in hospitalised patients: a consensus from the Antimicrobial Stewardship and Resistance Working Groups of the International Society of Chemotherapy. Int J Antimicrob Agents. 2016;48(3):239-46.

7. Halaby T, Al Naiemi N, Beishuizen B, et al. Impact of single room design on the spread of multi-drug resistant bacteria in an intensive care unit. Antimicrob Resist Infect Control. 2017;6:117.

8. Türe Yüce Z, Alp E. Infection Control Bundles for the Prevention of Hospital Infections Mediterr J Infect Microb Antimicrob. 2016;5:8

9. Anwar MM, Elareed HR. Improvement of hand hygiene compliance among health care workers in intensive care units. J Prev Med Hyg. 2019;60(1): E31-E35.

10. Simon M, Maben J, Murrells T, Griffiths P. Is single room hospital accommodation associated with differences in healthcare-associated infection, falls, pressure ulcers or medication errors? A natural experiment with non-equivalent controls. J Health Serv Res Policy. 2016;21(3):147-155.

11. Zingg W, Holmes A, Dettenkofer M, et al. Hospital organisation, management, and structure for prevention of healthcare-associated infection: a systematic review and expert consensus. Lancet Infect Dis. 2015;15(2):212-24.

12. Fox C, Wavra T, Drake DA, et al. Use of a Patient Hand Hygiene Protocol to Reduce Hospital-Acquired Infections and Improve Nurses' Hand Washing. Am J Crit Care.2015; 24 (3): 216-224.

13. Haverstick S, Goodrich C, Freeman R, James S, Kullar R, Ahrens M. Patients' Hand Washing and Reducing Hospital-Acquired Infection. Crit Care Nurse 2017; 37 (3): e1-e8.

14. Magalhães AMM, Costa DG, Riboldi CO, Mergen T, Barbosa AS, Moura GMSS. Association between workload of the nursing staff and patient safety outcomes. Rev Esc Enferm USP. 2017;51:e03255

15. Hugonnet S, Chevrolet JC, Pittet D. The effect of workload on infection risk in critically ill patients. Crit Care Med. 2007;35(1):76-81.

16. Di Muzio M, Cammilletti V, Petrelli E, Di Simone E. Hand hygiene in preventing nosocomial infections: a nursing research. Ann Ig. 2015;27(2):485-91.

17. Teltsch DY, Hanley J, Loo V, Goldberg P, Gursahaney A, Buckeridge DL. Infection Acquisition Following Intensive Care Unit Room Privatisation. Arch Intern Med. 2011;171(1):32-38.

18. Barbadoro P, Labricciosa FM, Recanatini C, Gori G, Tirabassi $F$, Martini E. Catheter-associated urinary tract infection: Role of the setting of catheter insertion. Am J Infect Control. 2015;43(7):707-10

19. Meddings J, Saint S, Krein SL, et al. Systematic Review of Interventions to Reduce Urinary Tract Infection in Nursing Home Residents. J Hosp Med. 2017;12(5):356-368.

20. Rosenthal VD, Bat-Erdene I, Gupta D, et al. International Nosocomial Infection Control Consortium (INICC) report, data summary of 45 countries for 2012-2017: Device-associated module. Am J Infect Control. 2019; . pii: S0196-6553(19)307953. doi: 10.1016/j.ajic.2019.08.023.

21. https://hsgm.saglik.gov.tr/depo/Duyurular/Surveyans Agi_Ozet_Raporu_2017/USHIESA_OZET_RAPOR_2017.pdf. Available date: 23.03 .2020

22. P Gunasekera, MBChB FRCA, A Gratrix, MBChB MEd FCARCSI FRCA FFICM, Ventilator-associated pneumonia, BJA Education, Volume 16, Issue 6, June 2016, Pages 198-202, https://doi. org/10.1093/bjaed/mkv046.

23. Khan $\mathrm{R}$, Al-Dorzi HM, Al-Attas $\mathrm{K}$ et al. The impact of implementing multifaceted interventions on the prevention of ventilator-associated pneumonia. Am J Infect Control. 2016;44(3):320-6. 
APPendix 1. The design OF the INTENSIVE CARE UNITS, INFECTION CARE PRACTICES AND STAFFING RESPONSIBILITIES IN THE ICUS OF THE OLDH VERSUS THE NeWH

\section{Design}

OldH: An open plan area containing thirteen beds. Plus, four separate isolation rooms each with one bed. Total number of beds: Seventeen.

NewH: Twenty-eight individual rooms, each with one bed.

\section{Infection control measures}

Standard infection control measures were applied in both the $\mathrm{OldH}$ and the $\mathrm{NewH}$ at all times.

Rectal screening for Vancomycin-resistant Enterococci (VRE) was performed on admission if the patient had been transferred from another hospital and after that weekly in both units.

Patients infected or colonised with multi-drug resistant microorganisms: contact isolation procedures were applied. In the $\mathrm{OldH}$, infected or colonised patients stayed in one of the four isolation rooms whenever possible.
Skin antiseptics were located next to the patient beds in the OldH.

Skin antiseptics were located next to the patient beds in the NewH and also at the exit from the patient's room.

Three washing-stands were located centrally in the open-plain area containing thirteen beds.

Twenty-eight washing-stands were located, one for each of the individual rooms.

\section{Environmental cleaning}

Environmental cleaning was performed three times a day in the OldH.

Environmental cleaning was performed twice a day in the $\mathrm{NewH}$.

\section{Staffing responsibilities}

OldH: Thirty-three nurses each worked a forty-eight hours per week shift system.

Each nurse was assigned to provide dedicated care for two patients in each shift.

NewH: Forty 40 nurses work in fifty-six hours per week shift system

Each nurse was assigned to provide dedicated care for two or three patients in each shift.

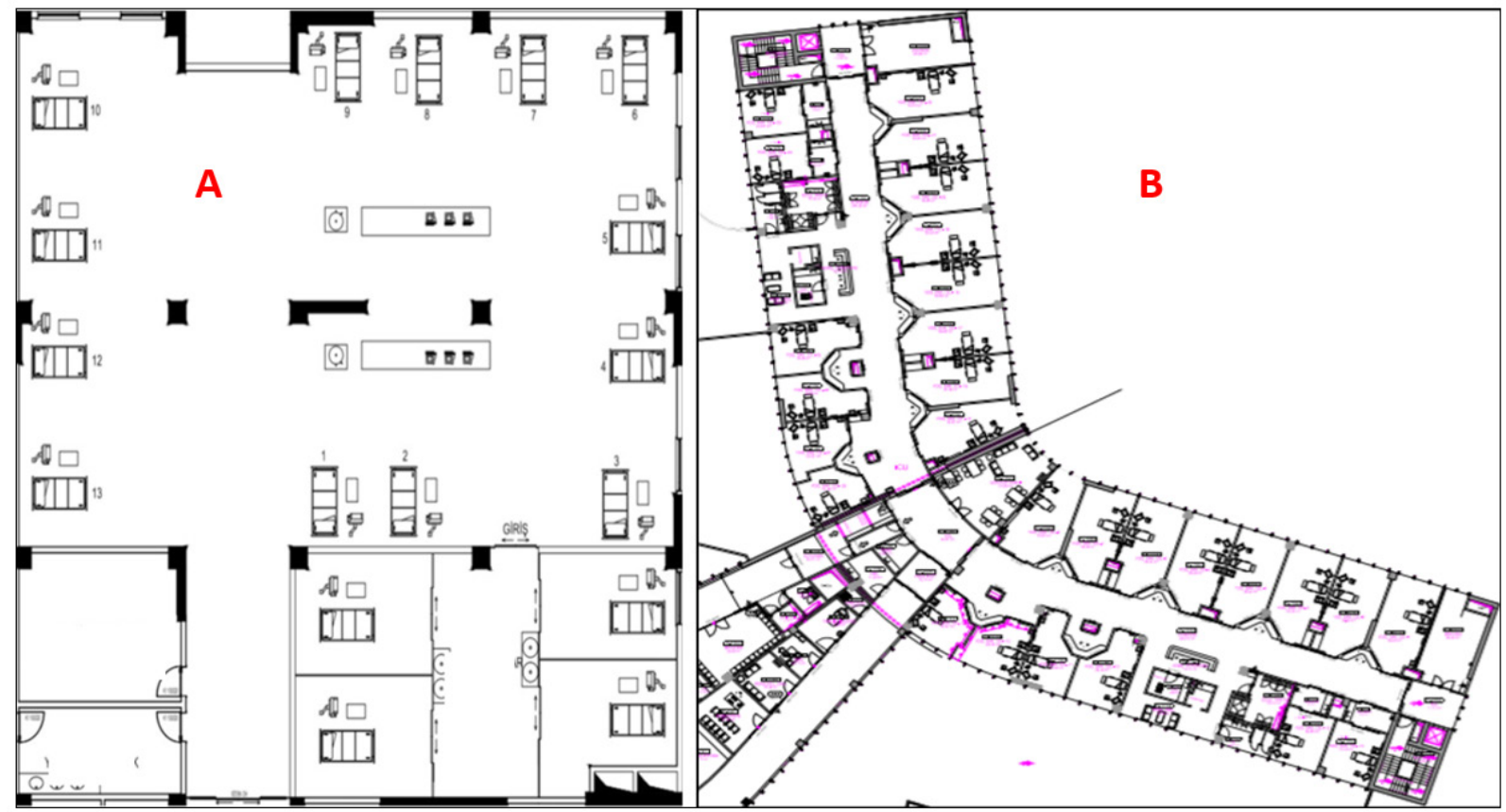

Supplementary figure 1: Intensive Care Unit Plans in the Old Hospital (A) and The New hospital (B). OldH: Total working area is $516 \mathrm{~m}^{2}$. NewH: Total working area is $1600 \mathrm{~m}^{2}$. 